\title{
On the periodic boundary value problem for Duffing type fractional differential equation with $p$-Laplacian operator
}

Hua Jin ${ }^{*}$ and Wenbin Liu

"Correspondence:

jinhua197927@163.com

Department of Mathematics, China

University of Mining and

Technology, Xuzhou, 221116,

PR China

\section{Springer}

\begin{abstract}
By using the continuation theorem of coincidence degree theory, we study the existence of solutions of Duffing type fractional differential equations with a $p$-Laplacian operator. Under certain nonlinear growth conditions of the nonlinearity, we obtain a new result on the existence of solutions.
\end{abstract}

MSC: 34A08; 34B15

Keywords: continuation theory; fractional differential equation; $p$-Laplacian operator; periodic boundary conditions

\section{Introduction}

Fractional calculus is a generalization of ordinary differentiation and integration on an arbitrary order that may be noninteger. Fractional differential equations have been proved to be valuable tools in the modeling of many phenomena in various fields of science and engineering. We can see numerous applications in viscoelasticity, neurons, electrochemistry, control, etc. (see [1-6]). Recently, with the intensive development of the theory of fractional calculus itself and its applications, there have many important results of fractional differential equations on initial value problems, and boundary value problems at nonresonance and resonance (see [7-12]).

In the study of the turbulent flow in a porous medium, Leibenson (see [13]) introduced the $p$-Laplacian equation as follows:

$$
\left(\phi_{p}\left(x^{\prime}(t)\right)\right)^{\prime}=f\left(t, x(t), x^{\prime}(t)\right)
$$

where $\phi_{p}(s)=|s|^{p-2} s, p>1$. Obviously, $\phi_{p}$ is invertible and its inverse operator is $\phi_{q}$, where $q>1$ is a constant such that $1 / p+1 / q=1$. In the past few decades, many important results relative to (1.1) with certain boundary value conditions have been obtained. See the papers [14-20] and the references therein. However, as far as we know, work on the existence of solutions for periodic boundary value problems (PBVPs for short) of fractional differential equations was discussed less.

(c) 2015 Jin and Liu. This article is distributed under the terms of the Creative Commons Attribution 4.0 International License (http://creativecommons.org/licenses/by/4.0/), which permits unrestricted use, distribution, and reproduction in any medium, provided you give appropriate credit to the original author(s) and the source, provide a link to the Creative Commons license, and indicate if changes were made. 
The aim of this paper is to concentrate on the following periodic boundary value problem for Duffing type fractional differential equations with $p$-Laplacian operator:

$$
\left\{\begin{array}{l}
D_{0^{+}}^{\beta}\left(\phi_{p}\left(D_{0^{+}}^{\alpha} x(t)\right)\right)+g(t, x(t))=e(t), \quad t \in[0, T] \\
x(0)=x(T), \quad D_{0^{+}}^{\alpha} x(0)=D_{0^{+}}^{\alpha} x(T)
\end{array}\right.
$$

where $0<\alpha, \beta \leq 1, D_{0^{+}}^{\alpha}, D_{0^{+}}^{\beta}$ are Caputo fractional derivatives, $T>0$ is a given constant, and $g:[0, T] \times \mathbb{R} \rightarrow \mathbb{R}, e:[0, T] \rightarrow \mathbb{R}$ are continuous. Throughout this paper, we assume that

$$
\int_{0}^{T}(T-s)^{\beta-1} e(s) d s=0
$$

The choice of periodic boundary conditions is motivated by the difficulty in the study of the PBVP

$$
\left\{\begin{array}{l}
D_{0^{+}}^{\beta}\left(\phi_{p}\left(D_{0^{+}}^{\alpha} x(t)\right)\right)=h(t), \quad t \in[0, T], \\
x(0)=x(T), \quad D_{0^{+}}^{\alpha} x(0)=D_{0^{+}}^{\alpha} x(T) .
\end{array}\right.
$$

As we know, PBVP (1.3) is not solvable for each $h \in C([0, T], \mathbb{R})$, and, when solvable, has no unique solution because $x(t)+c, \forall c \in \mathbb{R}$ is a solution together with $x(t)$. A trivial necessary condition for the solvability of PBVP (1.3) is that $\int_{0}^{T}(T-s)^{\beta-1} h(s) d s=0$.

Notice that $D_{0^{+}}^{\beta}\left(\phi_{p}\left(D_{0^{+}}^{\alpha}\right)\right)$ is a nonlinear operator, so the coincidence degree theory for linear differential operators is invalid in the direct application to it.

The rest of this paper is organized as follows. In Section 2, we describe the fractional differential operator and some lemmas. In Section 3, some sufficient conditions for the existence of solutions for PBVP (1.2) are established, and a new result on the existence of solutions is obtained. Finally, in Section 4, an example is given to illustrate the main result.

\section{Preliminaries}

Some definitions of the fractional derivative have emerged over the years (see [21, 22]), and in this paper we restrict our attention to the use of the Caputo fractional derivative. In this section, we introduce some basic definitions and lemmas which will be used in what follows. For details, we refer the reader to [21-25].

Definition 2.1 The Riemann-Liouville fractional integral operator of order $\alpha>0$ of a function $u:(0,+\infty) \rightarrow \mathbb{R}$ is given by

$$
I_{0^{+}}^{\alpha} u(t)=\frac{1}{\Gamma(\alpha)} \int_{0}^{t}(t-s)^{\alpha-1} u(s) d s
$$

provided that the right side integral is pointwise defined on $(0,+\infty)$, where $\Gamma(\cdot)>0$ is the Gamma function.

Definition 2.2 The Caputo fractional derivative of order $\alpha>0$ of a continuous function $u:(0,+\infty) \rightarrow \mathbb{R}$ is given by

$$
D_{0^{+}}^{\alpha} u(t)=I_{0^{+}}^{n-\alpha} \frac{d^{n} u(t)}{d t^{n}}=\frac{1}{\Gamma(n-\alpha)} \int_{0}^{t}(t-s)^{n-\alpha-1} u^{(n)}(s) d s,
$$


where $n$ is the smallest integer greater than or equal to $\alpha$, provided that the right side integral is pointwise defined on $(0,+\infty)$.

Lemma 2.1 (see [23]) Let $\alpha>0$. Assume that $u, D_{0^{+}}^{\alpha} u \in L([0, T], \mathbb{R})$. Then the following equality holds:

$$
I_{0^{+}}^{\alpha} D_{0^{+}}^{\alpha} u(t)=u(t)+c_{0}+c_{1} t+\cdots+c_{n-1} t^{n-1},
$$

where $c_{i} \in \mathbb{R}, i=0,1, \ldots, n-1$, here $n$ is the smallest integer greater than or equal to $\alpha$.

Lemma 2.2 (see [24]) For any $u, v \geq 0$,

$$
\phi_{p}(u+v) \leq \begin{cases}\phi_{p}(u)+\phi_{p}(v), & \text { if } p<2 ; \\ 2^{p-2}\left(\phi_{p}(u)+\phi_{p}(v)\right), & \text { if } p \geq 2 .\end{cases}
$$

Now we briefly recall some notations and an abstract existence result, which can be found in [25].

Let $X, Y$ be real Banach spaces, $L: \operatorname{dom} L \subset X \rightarrow Y$ be a Fredholm operator with index zero, and $P: X \rightarrow X, Q: Y \rightarrow Y$ be projectors such that

$$
\begin{aligned}
& \operatorname{Im} P=\operatorname{Ker} L, \quad \operatorname{Ker} Q=\operatorname{Im} L, \\
& X=\operatorname{Ker} L \oplus \operatorname{Ker} P, \quad Y=\operatorname{Im} L \oplus \operatorname{Im} Q .
\end{aligned}
$$

It follows that

$$
\left.L\right|_{\operatorname{dom} L \cap \operatorname{Ker} P}: \operatorname{dom} L \cap \operatorname{Ker} P \rightarrow \operatorname{Im} L
$$

is invertible. We denote the inverse by $K_{P}$.

If $\Omega$ is an open bounded subset of $X$ such that $\operatorname{dom} L \cap \bar{\Omega} \neq \varnothing$, then the map $N: X \rightarrow Y$ will be called $L$-compact on $\bar{\Omega}$ if $Q N(\bar{\Omega})$ is bounded and $K_{P}(I-Q) N: \bar{\Omega} \rightarrow X$ is compact.

Lemma 2.3 (see [25]) Let $X$ and $Y$ be two Banach spaces, $L: \operatorname{dom} L \subset X \rightarrow Y$ be a Fredholm operator with index zero, $\Omega \subset X$ be an open bounded set, and $N: \bar{\Omega} \rightarrow Y$ be Lcompact on $\bar{\Omega}$. Suppose that all of the following conditions hold:

(1) $L x \neq \lambda N x, \forall x \in \partial \Omega \cap \operatorname{dom} L, \lambda \in(0,1)$;

(2) $Q N x \neq 0, \forall x \in \partial \Omega \cap \operatorname{Ker} L$;

(3) $\operatorname{deg}(J Q N, \Omega \cap \operatorname{Ker} L, 0) \neq 0$, where $J: \operatorname{Im} Q \rightarrow \operatorname{Ker} L$ is an isomorphism map.

Then the equation $L x=N x$ has at least one solution on $\bar{\Omega} \cap \operatorname{dom} L$.

\section{Existence result}

For making use of the continuation theorem to study the existence of solutions for PBVP (1.2), we consider a system as follows:

$$
\left\{\begin{array}{l}
D_{0^{+}}^{\alpha} x_{1}(t)=\phi_{q}\left(x_{2}(t)\right), \\
D_{0^{+}}^{\beta} x_{2}(t)=e(t)-g\left(t, x_{1}(t)\right), \\
x_{1}(0)=x_{1}(T), \quad x_{2}(0)=x_{2}(T) .
\end{array}\right.
$$


Clearly, if $x(\cdot)=\left(x_{1}(\cdot), x_{2}(\cdot)\right)^{\mathrm{T}}$ is a solution of PBVP $(3.1)$, then $x_{1}(\cdot)$ must be a solution of PBVP (1.2). So, to prove PBVP (1.2) has solutions, we only need to show that PBVP (3.1) has solutions.

In this paper, we take $X=\left\{x=\left(x_{1}, x_{2}\right)^{\mathrm{T}} \mid x_{1}, x_{2} \in C([0, T], \mathbb{R})\right\}$ with the norm $\|x\|=$ $\max \left\{\left\|x_{1}\right\|_{0},\left\|x_{2}\right\|_{0}\right\}$, where $\left\|x_{i}\right\|_{0}=\max _{t \in[0, T]}\left|x_{i}(t)\right|(i \in\{1,2\})$. By means of the linear functional analysis theory, we can prove $X$ is a Banach space.

Define the operator $L: \operatorname{dom} L \subset X \rightarrow X$ by

$$
L x=\left(\begin{array}{c}
D_{0^{+}}^{\alpha} x_{1} \\
D_{0^{+}}^{\beta} x_{2}
\end{array}\right)
$$

where

$$
\begin{aligned}
\operatorname{dom} L= & \left\{x \in X \mid D_{0^{+}}^{\alpha} x_{1}, D_{0^{+}}^{\beta} x_{2} \in C([0, T], \mathbb{R}),\right. \\
& \left.x_{1}(0)=x_{1}(T), x_{2}(0)=x_{2}(T)\right\} .
\end{aligned}
$$

Let $N: X \rightarrow X$ be defined by

$$
N x(t)=\left(\begin{array}{c}
\phi_{q}\left(x_{2}(t)\right) \\
e(t)-g\left(t, x_{1}(t)\right)
\end{array}\right), \quad \forall t \in[0, T] .
$$

It is easy to see that PBVP (3.1) can be converted to the operator equation

$$
L x=N x, \quad x \in \operatorname{dom} L .
$$

Now let us introduce some lemmas.

Lemma 3.1 Let L be defined by (3.2), then

$$
\begin{aligned}
& \operatorname{Ker} L=\left\{x \in X \mid x(t)=c, \forall t \in[0, T], c \in \mathbb{R}^{2}\right\} \text {, } \\
& \left.\operatorname{Im} L=\left\{\begin{array}{l|l}
y \in X & \left(\begin{array}{l}
\int_{0}^{T}(T-s)^{\alpha-1} y_{1}(s) d s \\
\int_{0}^{T}(T-s)^{\beta-1} y_{2}(s) d s
\end{array}\right.
\end{array}\right)=0\right\} .
\end{aligned}
$$

Proof Obviously, from Lemma 2.1, we can see that (3.4) holds.

If $y \in \operatorname{Im} L$, then there exists $x \in \operatorname{dom} L$ such that $y=L x$. That is, $y_{1}(t)=D_{0^{+}}^{\alpha} x_{1}(t), y_{2}(t)=$ $D_{0^{+}}^{\beta} x_{2}(t)$. By using Lemma 2.1, we have

$$
\begin{array}{ll}
x_{1}(t)=c_{1}+\frac{1}{\Gamma(\alpha)} \int_{0}^{t}(t-s)^{\alpha-1} y_{1}(s) d s, & c_{1} \in \mathbb{R}, \\
x_{2}(t)=c_{2}+\frac{1}{\Gamma(\beta)} \int_{0}^{t}(t-s)^{\beta-1} y_{2}(s) d s, & c_{2} \in \mathbb{R} .
\end{array}
$$

From conditions $x_{1}(0)=x_{1}(T), x_{2}(0)=x_{2}(T)$, we obtain

$$
\left(\begin{array}{l}
\int_{0}^{T}(T-s)^{\alpha-1} y_{1}(s) d s \\
\int_{0}^{T}(T-s)^{\beta-1} y_{2}(s) d s
\end{array}\right)=0 .
$$


On the other hand, suppose $y \in X$ and satisfies (3.6). Let $x_{1}(t)=I_{0^{+}}^{\alpha} y_{1}(t), x_{2}(t)=I_{0^{+}}^{\beta} y_{2}(t)$. Obviously $x_{1}(0)=x_{1}(T), x_{2}(0)=x_{2}(T)$. Hence $x=\left(x_{1}, x_{2}\right)^{\mathrm{T}} \in \operatorname{dom} L$ and $L x=y$. So $y \in \operatorname{Im} L$. The proof is complete.

Lemma 3.2 Let $L$ be defined by (3.2), then $L$ is a Fredholm operator of index zero. The projectors $P: X \rightarrow X$ and $Q: X \rightarrow X$ can be defined as

$$
\begin{aligned}
P x(t) & =x(0), \quad \forall t \in[0, T], \\
Q y(t) & =\left(\begin{array}{c}
\frac{\alpha}{T^{\alpha}} \int_{0}^{T}(T-s)^{\alpha-1} y_{1}(s) d s \\
\frac{\beta}{T^{\beta}} \int_{0}^{T}(T-s)^{\beta-1} y_{2}(s) d s
\end{array}\right):=\left(\begin{array}{l}
(Q y)_{1} \\
(Q y)_{2}
\end{array}\right), \quad \forall t \in[0, T] .
\end{aligned}
$$

Furthermore, the operator $K_{P}: \operatorname{Im} L \rightarrow \operatorname{dom} L \cap \operatorname{Ker} P$ can be written as

$$
K_{P} y=\left(\begin{array}{c}
I_{0^{+}}^{\alpha} y_{1} \\
I_{0^{+}}^{\beta} y_{2}
\end{array}\right)
$$

which is $\left(\left.L\right|_{\operatorname{dom} L \cap \operatorname{Ker} P}\right)^{-1}$.

Proof For any $y \in X$, we have

$$
Q^{2} y=Q\left(\begin{array}{c}
(Q y)_{1} \\
(Q y)_{2}
\end{array}\right)=\left(\begin{array}{l}
(Q y)_{1} \frac{\alpha}{T^{\alpha}} \int_{0}^{T}(T-s)^{\alpha-1} d s \\
(Q y)_{2} \frac{\beta}{T^{\beta}} \int_{0}^{T}(T-s)^{\beta-1} d s
\end{array}\right)=Q y .
$$

Let $y^{*}=y-Q y=\left(\begin{array}{c}y_{1}^{*} \\ y_{2}^{*}\end{array}\right)$, then we get

$$
\begin{aligned}
\int_{0}^{T}(T-s)^{\alpha-1} y_{1}^{*}(s) d s & =\int_{0}^{T}(T-s)^{\alpha-1} y_{1}(s) d s-\int_{0}^{T}(T-s)^{\alpha-1}(Q y)_{1} d s \\
& =\frac{T^{\alpha}}{\alpha}\left((Q y)_{1}-\left(Q^{2} y\right)_{1}\right)=0 .
\end{aligned}
$$

Similarly, we have $\int_{0}^{T}(T-s)^{\beta-1} y_{2}^{*}(s) d s=0$. So $y^{*} \in \operatorname{Im} L$. Hence $X=\operatorname{Im} L+\operatorname{Im} Q$. Since $\operatorname{Im} L \cap \operatorname{Im} Q=\{0\}$, we have $X=\operatorname{Im} L \oplus \operatorname{Im} Q$. Thus

$$
\operatorname{dim} \operatorname{Ker} L=\operatorname{dim} \operatorname{Im} Q=\operatorname{codim} \operatorname{Im} L=2 .
$$

This means that $L$ is a Fredholm operator of index zero.

From the definition of $K_{P}$, for $y \in \operatorname{Im} L$, we have

$$
L K_{P} y=\left(\begin{array}{c}
D_{0^{+}}^{\alpha} I_{0^{+}}^{\alpha} y_{1} \\
D_{0^{+}}^{\beta} I_{0^{+}}^{\beta} y_{2}
\end{array}\right)=y .
$$

On the other hand, for $x \in \operatorname{dom} L \cap \operatorname{Ker} P$, we have $x_{1}(0)=x_{2}(0)=0$. By Lemma 2.1, we get

$$
K_{P} L x=\left(\begin{array}{l}
x_{1}-x_{1}(0) \\
x_{2}-x_{2}(0)
\end{array}\right)=x
$$

So, we know that $K_{P}=\left(L_{\operatorname{dom} L \cap \operatorname{Ker} P}\right)^{-1}$. The proof is complete. 
Lemma 3.3 Let $N$ be defined by (3.3). Assume $\Omega \subset X$ is an open bounded subset such that $\operatorname{dom} L \cap \bar{\Omega} \neq \varnothing$, then $N$ is L-compact on $\bar{\Omega}$.

Proof By the continuity of $\phi_{q}, e, g$, we find that $Q N(\bar{\Omega})$ and $K_{P}(I-Q) N(\bar{\Omega})$ are bounded. Moreover, there exists a constant $M>0$ such that $\|(I-Q) N x\| \leq M, \forall x \in \bar{\Omega}, t \in[0, T]$. Thus, in view of the Arzelà-Ascoli theorem, we only need prove that $K_{P}(I-Q) N(\bar{\Omega}) \subset X$ is equicontinuous.

For $0 \leq t_{1}<t_{2} \leq T, x \in \bar{\Omega}$, we have

$$
\begin{aligned}
K_{P}(I-Q) N x\left(t_{2}\right)-K_{P}(I-Q) N x\left(t_{1}\right) \\
\quad=\left(\begin{array}{c}
I_{0^{+}}^{\alpha}((I-Q) N x)_{1}\left(t_{2}\right)-I_{0^{+}}^{\alpha}((I-Q) N x)_{1}\left(t_{1}\right) \\
I_{0^{+}}^{\beta}((I-Q) N x)_{2}\left(t_{2}\right)-I_{0^{+}}^{\beta}((I-Q) N x)_{2}\left(t_{1}\right)
\end{array}\right) .
\end{aligned}
$$

From $\|(I-Q) N x\| \leq M, \forall x \in \bar{\Omega}, t \in[0, T]$, we can see that

$$
\begin{aligned}
& \left|\frac{1}{\Gamma(\alpha)}\left(\int_{0}^{t_{2}}\left(t_{2}-s\right)^{\alpha-1}((I-Q) N x)_{1}(s) d s-\int_{0}^{t_{1}}\left(t_{1}-s\right)^{\alpha-1}((I-Q) N x)_{1}(s) d s\right)\right| \\
& \quad \leq \frac{M}{\Gamma(\alpha)}\left(\int_{0}^{t_{1}}\left(\left(t_{1}-s\right)^{\alpha-1}-\left(t_{2}-s\right)^{\alpha-1}\right) d s+\int_{t_{1}}^{t_{2}}\left(t_{2}-s\right)^{\alpha-1} d s\right) \\
& \quad=\frac{M}{\Gamma(\alpha+1)}\left(t_{1}^{\alpha}-t_{2}^{\alpha}+2\left(t_{2}-t_{1}\right)^{\alpha}\right) \\
& \quad \leq \frac{M}{\Gamma(\alpha+1)}\left(t_{2}^{\alpha}-t_{1}^{\alpha}+2\left(t_{2}-t_{1}\right)^{\alpha}\right) .
\end{aligned}
$$

Since $t^{\alpha}$ is uniformly continuous on $[0, T]$, we find that $\left(K_{P}(I-Q) N(\bar{\Omega})\right)_{1} \subset C([0, T], \mathbb{R})$ is equicontinuous. A similar proof can show that $\left(K_{P}(I-Q) N(\bar{\Omega})\right)_{2} \subset C([0, T], \mathbb{R})$ is equicontinuous. So we find that $K_{P}(I-Q) N: \bar{\Omega} \rightarrow X$ is compact. The proof is complete.

Now we give the main result as regards the existence of solutions for PBVP (1.2).

\section{Theorem 3.1 Assume that:}

$\left(\mathrm{H}_{1}\right)$ there exists a constant $d_{1}>0$ such that

$$
(-1)^{i} x g(t, x)>0 \quad(i \in\{1,2\}), \forall t \in[0, T],|x|>d_{1}
$$

$\left(\mathrm{H}_{2}\right)$ there exist a constant $d_{2}>0$ and nonnegative functions $a, b \in C([0, T], \mathbb{R})$ such that

$$
|g(t, x)| \leq a(t)|x|^{p-1}+b(t), \quad \forall t \in[0, T],|x|>d_{2} .
$$

Then PBVP (1.2) has at least one solution, provided that

$$
\begin{aligned}
& \gamma_{1}:=\frac{2^{p} T^{\beta+\alpha p-\alpha}\|a\|_{0}}{\Gamma(\beta+1)(\Gamma(\alpha+1))^{p-1}}<1, \quad \text { if } p<2 \\
& \gamma_{2}:=\frac{2^{2 p-2} T^{\beta+\alpha p-\alpha}\|a\|_{0}}{\Gamma(\beta+1)(\Gamma(\alpha+1))^{p-1}}<1, \quad \text { if } p \geq 2 .
\end{aligned}
$$




\section{Proof Set}

$$
\Omega^{\prime}=\{x \in \operatorname{dom} L \mid L x=\lambda N x, \lambda \in(0,1)\} .
$$

For $x \in \Omega^{\prime}$, we get $N x \in \operatorname{Im} L$. So by (3.5), we have

$$
\begin{aligned}
& \int_{0}^{T}(T-s)^{\alpha-1} \phi_{q}\left(x_{2}(s)\right) d s=0 \\
& \int_{0}^{T}(T-s)^{\beta-1}\left(e(s)-g\left(s, x_{1}(s)\right)\right) d s=0 .
\end{aligned}
$$

From the integral mean value theorem and $\int_{0}^{T}(T-s)^{\beta-1} e(s) d s=0$, there exist constants $\zeta, \eta \in(0, T)$ such that $x_{2}(\zeta)=0, g\left(\eta, x_{1}(\eta)\right)=0$. Together with the condition $\left(\mathrm{H}_{1}\right)$, we have $\left|x_{1}(\eta)\right| \leq d_{1}$. By Lemma 2.1, we have

$$
x_{1}(t)=x_{1}(\eta)-I_{0^{+}}^{\alpha} D_{0^{+}}^{\alpha} x_{1}(\eta)+I_{0^{+}}^{\alpha} D_{0^{+}}^{\alpha} x_{1}(t)
$$

which, together with

$$
\begin{aligned}
\left|I_{0^{+}}^{\alpha} D_{0^{+}}^{\alpha} x_{1}(t)\right| & =\frac{1}{\Gamma(\alpha)}\left|\int_{0}^{t}(t-s)^{\alpha-1} D_{0^{+}}^{\alpha} x_{1}(s) d s\right| \\
& \leq \frac{1}{\Gamma(\alpha)}\left\|D_{0^{+}}^{\alpha} x_{1}\right\|_{0} \cdot \frac{1}{\alpha} t^{\alpha} \\
& \leq \frac{T^{\alpha}}{\Gamma(\alpha+1)}\left\|D_{0^{+}}^{\alpha} x_{1}\right\|_{0^{\prime}}, \quad \forall t \in[0, T],
\end{aligned}
$$

and $\left|x_{1}(\eta)\right| \leq d_{1}$, yields

$$
\left\|x_{1}\right\|_{0} \leq d_{1}+\frac{2 T^{\alpha}}{\Gamma(\alpha+1)}\left\|D_{0^{+}}^{\alpha} x_{1}\right\|_{0} .
$$

On the other hand, if $x \in \Omega^{\prime}$, we have

$$
\left\{\begin{array}{l}
D_{0^{+}}^{\alpha} x_{1}(t)=\lambda \phi_{q}\left(x_{2}(t)\right) \\
D_{0^{+}}^{\beta} x_{2}(t)=\lambda\left(e(t)-g\left(t, x_{1}(t)\right)\right) .
\end{array}\right.
$$

From the first equation of (3.9), we get $x_{2}(t)=\phi_{p}\left(\lambda^{-1} D_{0^{+}}^{\alpha} x_{1}(t)\right)$. By substituting it into the second equation of (3.9), we get

$$
D_{0^{+}}^{\beta}\left(\phi_{p}\left(D_{0^{+}}^{\alpha} x_{1}(t)\right)\right)=\lambda^{p} e(t)-\lambda^{p} g\left(t, x_{1}\right):=\lambda^{p} N_{g} x_{1}(t) .
$$

Thus, by Lemma 2.1, we obtain

$$
\phi_{p}\left(D_{0^{+}}^{\alpha} x_{1}(t)\right)=c_{0}+\lambda^{p} I_{0^{+}}^{\beta} N_{g} x_{1}(t) .
$$

Then we have

$$
x_{1}(t)=c_{1}+I_{0^{+}}^{\alpha} \phi_{q}\left(c_{0}+\lambda^{p} I_{0^{+}}^{\beta} N_{g} x_{1}\right)(t)
$$


By the boundary condition $x_{1}(0)=x_{1}(T)$, we get

$$
\frac{1}{\Gamma(\alpha)} \int_{0}^{T}(T-s)^{\alpha-1} \phi_{q}\left(c_{0}+\lambda^{p} I_{0^{+}}^{\beta} N_{g} x_{1}(s)\right) d s=0 .
$$

Obviously, there exists a constant $\xi \in(0, T)$ such that $\phi_{q}\left(c_{0}+\lambda^{p} I_{0^{+}}^{\beta} N_{g} x_{1}(\xi)\right)=0$, which implies that $c_{0}=-\lambda^{p} I_{0^{+}}^{\beta} N_{g} x_{1}(\xi)$. By substituting it into (3.10), we have

$$
\phi_{p}\left(D_{0^{+}}^{\alpha} x_{1}(t)\right)=-\lambda^{p} I_{0^{+}}^{\beta} N_{g} x_{1}(\xi)+\lambda^{p} I_{0^{+}}^{\beta} N_{g} x_{1}(t) .
$$

From the hypothesis $\left(\mathrm{H}_{2}\right)$, we get

$$
\begin{aligned}
\left|I_{0^{+}}^{\beta} N_{g} x_{1}(t)\right| & =\frac{1}{\Gamma(\beta)}\left|\int_{0}^{t}(t-s)^{\beta-1} e(s) d s-\int_{0}^{t}(t-s)^{\beta-1} g\left(s, x_{1}(s)\right) d s\right| \\
& \leq \frac{T^{\beta}}{\Gamma(\beta+1)}\|e\|_{0}+\frac{1}{\Gamma(\beta)} \int_{0}^{t}(t-s)^{\beta-1}\left|g\left(s, x_{1}(s)\right)\right| d s \\
& \leq \frac{T^{\beta}}{\Gamma(\beta+1)}\left(\|e\|_{0}+\|a\|_{0}\left\|x_{1}\right\|_{0}^{p-1}+\|b\|_{0}+G_{d_{2}}\right), \quad \forall t \in[0, T],
\end{aligned}
$$

where $G_{d_{2}}=\max \left\{|g(t, x)||t \in[0, T]| x \mid, \leq d_{2}\right\}$. Together with (3.8), (3.11), and

$$
\left|\phi_{p}\left(D_{0^{+}}^{\alpha} x_{1}(t)\right)\right|=\left|D_{0^{+}}^{\alpha} x_{1}(t)\right|^{p-1}
$$

we have

$$
\begin{aligned}
\left\|D_{0^{+}}^{\alpha} x_{1}\right\|_{0}^{p-1} & \leq \frac{2 T^{\beta}}{\Gamma(\beta+1)}\left(\|e\|_{0}+\|a\|_{0}\left\|x_{1}\right\|_{0}^{p-1}+\|b\|_{0}+G_{d_{2}}\right) \\
& \leq \frac{2 T^{\beta}}{\Gamma(\beta+1)}\left[\|e\|_{0}+\|b\|_{0}+G_{d_{2}}+\|a\|_{0}\left(d_{1}+\frac{2 T^{\alpha}}{\Gamma(\alpha+1)}\left\|D_{0^{+}}^{\alpha} x_{1}\right\|_{0}\right)^{p-1}\right] .
\end{aligned}
$$

If $p<2$, by using Lemma 2.2 , we get

$$
\begin{aligned}
\left\|D_{0^{+}}^{\alpha} x_{1}\right\|_{0}^{p-1} \leq & \frac{2 T^{\beta}}{\Gamma(\beta+1)}\left(\|e\|_{0}+\|b\|_{0}+G_{d_{2}}\right) \\
& +\frac{2 T^{\beta}\|a\|_{0}}{\Gamma(\beta+1)}\left(d_{1}^{p-1}+\frac{\left(2 T^{\alpha}\right)^{p-1}}{(\Gamma(\alpha+1))^{p-1}}\left\|D_{0^{+}}^{\alpha} x_{1}\right\|_{0}^{p-1}\right) \\
= & A_{1}+\frac{2^{p} T^{\beta+\alpha p-\alpha}\|a\|_{0}}{\Gamma(\beta+1)(\Gamma(\alpha+1))^{p-1}}\left\|D_{0^{+}}^{\alpha} x_{1}\right\|_{0}^{p-1},
\end{aligned}
$$

where $A_{1}=\frac{2 T^{\beta}}{\Gamma(\beta+1)}\left(\|e\|_{0}+\|b\|_{0}+G_{d_{2}}\right)+\frac{2 T^{\beta}\|a\|_{0}}{\Gamma(\beta+1)} d_{1}^{p-1}$. Then, from (3.7), we have

$$
\left\|D_{0^{+}}^{\alpha} x_{1}\right\|_{0} \leq\left(\frac{A_{1}}{1-\gamma_{1}}\right)^{q-1}:=M_{1}
$$

Thus, from (3.8), we get

$$
\left\|x_{1}\right\|_{0} \leq d_{1}+\frac{2 T^{\alpha}}{\Gamma(\alpha+1)} M_{1}
$$


If $p \geq 2$, similar to the above argument, let $A_{2}=\frac{2 T^{\beta}}{\Gamma(\beta+1)}\left(\|e\|_{0}+\|b\|_{0}+G_{d_{2}}\right)+\frac{2^{p-1} T^{\beta}\|a\|_{0}}{\Gamma(\beta+1)} d_{1}^{p-1}$, we obtain

$$
\left\|x_{1}\right\|_{0} \leq d_{1}+\frac{2 T^{\alpha}}{\Gamma(\alpha+1)} M_{2}
$$

where $M_{2}=\left(\frac{A_{2}}{1-\gamma_{2}}\right)^{q-1}$. So, combining (3.12) with (3.13), we get

$$
\left\|x_{1}\right\|_{0} \leq \max \left\{d_{1}+\frac{2 T^{\alpha}}{\Gamma(\alpha+1)} M_{1}, d_{1}+\frac{2 T^{\alpha}}{\Gamma(\alpha+1)} M_{2}\right\}:=M .
$$

From the second equation of (3.9) and Lemma 2.1, we have

$$
x_{2}(t)=c+\lambda I_{0^{+}}^{\beta} N_{g} x_{1}(t)
$$

which together with $x_{2}(\zeta)=0$ yields

$$
x_{2}(t)=-\lambda I_{0^{+}}^{\beta} N_{g} x_{1}(\zeta)+\lambda I_{0^{+}}^{\beta} N_{g} x_{1}(t) .
$$

Then we have

$$
\left\|x_{2}\right\|_{0} \leq \frac{2 T^{\beta}}{\Gamma(\beta+1)}\left(\|e\|_{0}+G_{M}\right):=\bar{M},
$$

where $G_{M}=\max \{|g(t, x)||t \in[0, T]| x \mid, \leq M\}$. Together with (3.14), we obtain

$$
\|x\|=\max \left\{\left\|x_{1}\right\|_{0},\left\|x_{2}\right\|_{0}\right\} \leq \max \{M, \bar{M}\}:=M_{0} .
$$

Let $\Omega=\left\{x \in X \mid\|x\|<M_{0}+1\right\}$. From the above argument, we know that the equation

$$
L x=\lambda N x, \quad \forall \lambda \in(0,1)
$$

has no solution on $\partial \Omega \cap \operatorname{dom} L$. So the condition (1) of Lemma 2.3 is satisfied. Next the other two conditions of Lemma 2.3 are to be verified.

For $x \in \operatorname{Ker} L$, we have $x_{1}(t)=c_{1}, x_{2}(t)=c_{2}, \forall t \in[0, T], c_{1}, c_{2} \in \mathbb{R}$. If $Q N x=0$, we obtain

$$
\begin{aligned}
& \int_{0}^{T}(T-s)^{\alpha-1} \phi_{q}\left(c_{2}\right) d s=0 \\
& \int_{0}^{T}(T-s)^{\beta-1} g\left(s, c_{1}\right) d s=0 .
\end{aligned}
$$

From the first equality, we get $c_{2}=0$. From the second equality and $\left(\mathrm{H}_{1}\right)$, we have $\left|c_{1}\right| \leq d_{1}$. So $\|x\|=\max \left\{\left|c_{1}\right|,\left|c_{2}\right|\right\} \leq d_{1}<M_{0}+1$. Then the condition (2) of Lemma 2.3 is satisfied.

Define the operators $J: \operatorname{Im} Q \rightarrow \operatorname{Ker} L$ by

$$
J\left(x_{1}, x_{2}\right)^{\mathrm{T}}=\left((-1)^{i+1} x_{2}, x_{1}\right)^{\mathrm{T}},
$$

and $F:[0,1] \times \bar{\Omega} \rightarrow X$ by

$$
F(\mu, x)=\mu x+(1-\mu) J Q N x=\left(\begin{array}{c}
\mu x_{1}+(-1)^{i}(1-\mu) \frac{\beta}{T^{\beta}} \int_{0}^{T}(T-s)^{\beta-1} g\left(s, x_{1}(s)\right) d s \\
\mu x_{2}+(1-\mu) \frac{\alpha}{T^{\alpha}} \int_{0}^{T}(T-s)^{\alpha-1} \phi_{q}\left(x_{2}(s)\right) d s
\end{array}\right),
$$


where $i \in\{1,2\}$. Let $x \in \operatorname{Ker} L$ satisfying $F(\mu, x)=0$, we get $x_{1}(t)=c_{1}, x_{2}(t)=c_{2}, \forall t \in[0, T]$, $c_{1}, c_{2} \in \mathbb{R}$, and

$$
\begin{aligned}
& \mu c_{1}+(-1)^{i}(1-\mu) \frac{\beta}{T^{\beta}} \int_{0}^{T}(T-s)^{\beta-1} g\left(s, c_{1}\right) d s=0, \\
& \mu c_{2}+(1-\mu) \phi_{q}\left(c_{2}\right)=0 .
\end{aligned}
$$

From (3.16), we get $c_{2}=0$ because $c_{2}$ and $\phi_{q}\left(c_{2}\right)$ have the same sign. From (3.15), if $\mu=0$, we get $\left|c_{1}\right| \leq d_{1}$ because of $\left(\mathrm{H}_{1}\right)$. If $\mu \in(0,1]$, we also get $\left|c_{1}\right| \leq d_{1}$. In fact, if $\left|c_{1}\right|>d_{1}$, in view of $\left(\mathrm{H}_{1}\right)$, one has

$$
\mu c_{1}^{2}+(1-\mu) \frac{\beta}{T^{\beta}} \int_{0}^{T}(T-s)^{\beta-1}(-1)^{i} c_{1} g\left(s, c_{1}\right) d s>0,
$$

which contradicts (3.15). From the argument above, we obtain $\|x\|<M_{0}+1$. Thus

$$
F(\mu, x) \neq 0, \quad \forall(\mu, x) \in[0,1] \times(\partial \Omega \cap \operatorname{Ker} L) .
$$

Hence, by the homotopy property of the degree, we have

$$
\begin{aligned}
\operatorname{deg}(J Q N, \Omega \cap \operatorname{Ker} L, 0) & =\operatorname{deg}(F(0, \cdot), \Omega \cap \operatorname{Ker} L, 0) \\
& =\operatorname{deg}(F(1, \cdot), \Omega \cap \operatorname{Ker} L, 0)=\operatorname{deg}(I, \Omega \cap \operatorname{Ker} L, 0) \neq 0 .
\end{aligned}
$$

So the condition (3) of Lemma 2.3 is satisfied.

Consequently, by using Lemma 2.3, the operator equation $L x=N x$ has at least one solution $x(\cdot)=\left(x_{1}(\cdot), x_{2}(\cdot)\right)^{\mathrm{T}}$ on $\bar{\Omega} \cap \operatorname{dom} L$. Namely, PBVP (1.2) has at least one solution $x_{1}(\cdot)$. The proof is complete.

\section{An example}

In this section, we will give an example to illustrate our main result.

Example 4.1 Consider the following PBVP for a fractional $p$-Laplacian equation:

$$
\left\{\begin{array}{l}
D_{0^{+}}^{\frac{3}{4}}\left(\phi_{4}\left(D_{0^{+}}^{\frac{1}{2}} x(t)\right)\right)-\frac{1}{120} x^{3}(t)+\frac{1}{2}=(1-t)^{\frac{1}{4}} \sin 2 \pi t, \quad t \in[0,1], \\
x(0)=x(1), \quad D_{0^{+}}^{\frac{1}{2}} x(0)=D_{0^{+}}^{\frac{1}{2}} x(1) .
\end{array}\right.
$$

Corresponding to PBVP (1.2), we get $p=4, \alpha=1 / 2, \beta=3 / 4, T=1, e(t)=(1-t)^{\frac{1}{4}} \sin 2 \pi t$, and

$$
g(t, x)=-\frac{1}{120} x^{3}+\frac{1}{2}
$$

Choose $a(t)=\frac{1}{120}, b(t)=1$. By a simple calculation, we obtain

$$
\begin{aligned}
& x g(t, x)=-\frac{x}{120}\left(x^{3}-60\right)<0, \quad \forall t \in[0,1],|x|>4, \\
& \gamma_{2}=\frac{2^{3} / 120}{\Gamma\left(\frac{3}{4}+1\right)}\left[\frac{2}{\Gamma\left(\frac{1}{2}+1\right)}\right]^{3}<1 .
\end{aligned}
$$




\section{Obviously, PBVP (4.1) satisfies all assumptions of Theorem 3.1. Hence, PBVP (4.1) has at least one solution.}

\section{Competing interests}

The authors declare that they have no competing interests.

\section{Authors' contributions}

All the authors contributed equally and significantly in writing this article. All the authors read and approved the final manuscript.

\section{Acknowledgements}

The authors would like to thank the anonymous referee for his/her valuable comments, which have improved the correctness and presentation of the manuscript. This work is supported by the National Natural Science Foundation of China (11271364).

Received: 10 October 2014 Accepted: 2 March 2015 Published online: 21 August 2015

\section{References}

1. Diethelm, K, Freed, AD: On the solution of nonlinear fractional order differential equations used in the modeling of viscoelasticity. In: Keil, F, Mackens, W, Voss, H, Werther, J (eds.) Scientific Computing in Chemical Engineering II-Computational Fluid Dynamics, Reaction Engineering and Molecular Properties, pp. 217-224. Springer, Heidelberg (1999)

2. Lundstrom, BN, Higgs, MH, Spain, WJ, Fairhall, AL: Fractional differentiation by neocortical pyramidal neurons. Nat. Neurosci. 11, 1335-1342 (2008)

3. Glockle, WG, Nonnenmacher, TF: A fractional calculus approach of self-similar protein dynamics. Biophys. J. 68, 46-53 (1995)

4. Hilfer, R: Applications of Fractional Calculus in Physics. World Scientific, Singapore (2000)

5. Mainardi, F: Fractional calculus: some basic problems in continuum and statistical mechanics. In: Carpinteri, A, Mainardi, F (eds.) Fractals and Fractional Calculus in Continuum Mechanics, pp. 291-348. Springer, Wien (1997)

6. Kirchner, JW, Feng, X, Neal, C: Fractal stream chemistry and its implications for contaminant transport in catchments. Nature 403, 524-526 (2000)

7. Babakhani, A, Gejji, VD: Existence of positive solutions of nonlinear fractional differential equations. J. Math. Anal. Appl. 278, 434-442 (2003)

8. Delbosco, D, Rodino, L: Existence and uniqueness for a nonlinear fractional differential equation. J. Math. Anal. Appl. 204, 609-625 (1996)

9. Kilbas, AA, Trujillo, JJ: Differential equations of fractional order: methods, results and problems-I. Appl. Anal. 78, 153-192 (2001)

10. Kilbas, AA, Trujillo, J: Differential equations of fractional order: methods, results and problems-II. Appl. Anal. 81, 435-493 (2002)

11. Agarwal, RP, O'Regan, D, Stanek, S: Positive solutions for Dirichlet problems of singular nonlinear fractional differential equations. J. Math. Anal. Appl. 371, 57-68 (2010)

12. Bai, Z: On solutions of some fractional $m$-point boundary value problems at resonance. Electron. J. Qual. Theory Differ. Equ. 2010, Article ID 37 (2010)

13. Leibenson, LS: General problem of the movement of a compressible fluid in a porous medium. Izv. Akad. Nauk Kirg. SSSR 9, 7-10 (1983) (in Russian)

14. Chen, T, Liu, W, Yang, C: Antiperiodic solutions for Liénard-type differential equation with p-Laplacian operator. Bound. Value Probl. 2010, Article ID 194824 (2010)

15. Jiang, D, Gao, W: Upper and lower solution method and a singular boundary value problem for the one-dimensional p-Laplacian. J. Math. Anal. Appl. 252, 631-648 (2000)

16. Lian, L, Ge, W: The existence of solutions of m-point $p$-Laplacian boundary value problems at resonance. Acta Math. Appl. Sin. 28, 288-295 (2005)

17. Liu, B, Yu, J: On the existence of solutions for the periodic boundary value problems with $p$-Laplacian operator. J. Syst. Sci. Math. Sci. 23, 76-85 (2003)

18. Pang, $\mathrm{H}, \mathrm{Ge}, \mathrm{W}$, Tian, M: Solvability of nonlocal boundary value problems for ordinary differential equation of higher order with a $p$-Laplacian. Comput. Math. Appl. 56, 127-142 (2008)

19. Su, H, Wang, B, Wei, Z, Zhang, X: Positive solutions of four-point boundary value problems for higher-order p-Laplacian operator. J. Math. Anal. Appl. 330, 836-851 (2007)

20. Zhang, J, Liu, W, Ni, J, Chen, T: Multiple periodic solutions of $p$-Laplacian equation with one-side Nagumo condition. J. Korean Math. Soc. 45, 1549-1559 (2008)

21. Podlubny, l: Fractional Differential Equations. Academic Press, San Diego (1999)

22. Samko, SG, Kilbas, AA, Marichev, Ol: Fractional Integrals and Derivatives: Theory and Applications. Gordon and Breach, Switzerland (1993)

23. Kilbas, AA, Srivastava, HM, Trujillo, JJ: Theory and Applications of Fractional Differential Equations. Elsevier, Amsterdam (2006)

24. Lian, $\mathrm{H}$ : Boundary value problems for nonlinear ordinary differential equations on infinite intervals, Doctoral thesis (2007)

25. Gaines, R, Mawhin, J: Coincidence Degree and Nonlinear Differential Equations. Springer, Berlin (1977) 